\title{
Computed Tomography-Guided Biopsy for Potts Disease: An Institutional Experience from an Endemic Developing Country
}

\author{
Muhammad Waqas, Mohsin Qadeer, Faizuddin Faiz, Mohammad Ali Alvi, Muhammad Ehsan Bari \\ Section of Neurosurgery, The Aga Khan University Hospital, Karachi, Pakistan
}

\section{Study Design: A retrospective chart review.}

Purpose: In endemic resource poor countries like Pakistan, most patients are diagnosed and treated for Potts disease on clinical and radiological grounds without a routine biopsy. The purpose of this study was to evaluate the use and effect of computed tomography (CT)-guided biopsy in the management of Potts disease since the technique is becoming increasingly available.

Overview of Literature: CT-guided biopsy of spinal lesions is routinely performed. Literature on the utility of the technique in endemic resource poor countries is little.

Methods: This study was conducted at the Neurosurgery section of Aga Khan University Hospital Karachi. All the patients with suspected Potts disease who underwent CT-guided biopsy during the 7 year period from 2007 to 2013 were included in this study. Details of the procedure, histopathology and microbiology were recorded.

Results: One hundred and seventy-eight patients were treated for suspected Potts disease during the study period. CT-guided biopsies of the spinal lesions were performed in 91 patients $(51.12 \%)$. Of the 91 procedures, $22(24.2 \%)$ were inconclusive because of inadequate sample (10), normal tissue (6) or reactive tissue (6). Sixty-nine biopsies were positive (75.8\%). Granulomatous inflammation was seen in 58 patients (84.05\%), positive acid-fast bacillus (AFB) smear in $4(5.7 \%)$ and positive AFB culture in 12 patients (17.3\%). All 91 cases in which CT-guided biopsy was performed responded positively to antituberculosis therapy (ATT).

Conclusions: $75.8 \%$ of the specimens yielded positive diagnoses. Granulomatous inflammation on histopathology was the commonest diagnostic feature. In this series, the rates of positive AFB smear and culture were low compared to previous literature.

Keywords: Potts disease; Computed tomography; Aspiration biopsy

\section{Introduction}

Tuberculosis is the second greatest cause of mortality worldwide due to a single infectious agent [1]. Almost a third of the entire population today has latent tuberculosis (TB), i.e., infected but asymptomatic. In 2012, 8.6 million people fell ill with TB and 1.3 million died from TB. Paki- stan ranks fifth among the high burden countries (HBCs) and fourth among multi drug resistant (MDR) HBCs and accounts for $61 \%$ of the TB burden in the World Health Organization Eastern Mediterranean Region [1]. The estimated prevalence of the disease is 350/100,000 and mortality is $33 / 100,000$ [2].

Potts disease or spinal tuberculosis is one of the most

Received Oct 7, 2014; Revised Nov 9, 2014; Accepted Nov 25, 2014

Corresponding author: Muhammad Ehsan Bari

Section of Neurosurgery, The Aga Khan University Hospital, Stadium Road Karachi, PO Box 3500, Karachi 74800, Pakistan

Tel: +92-3323475239, Fax: +92-2134864764, E-mail: ehsan.bari@aku.edu 
crippling manifestations of extra-pulmonary tuberculosis. Approximately $10 \%$ of all extra-pulmonary cases of tuberculosis have skeletal involvement [3] and the spine is affected in half of these patients [4]. The lesion most frequently involves the thoracic spine $(45 \%-50 \%)$ and the lumbar spine (40\%-45\%) [5]. The spine is usually affected through lymphatic spreading of the bacteria when the primary site might have turned quiescent [6] or from hematogenous seeding [7].

Though the clinical presentation may be highly variable depending on the site of the lesion [7], back pain is a consistent finding. Other symptoms include systemic manifestations (weight loss, fatigue, fever and malaise), neurological symptoms (weakness, paresthesia, numbness, bowel symptoms) [5,7-9] and structural deformity (gibbus, kyphosis) [5,8]. Laboratory investigations include deranged erythrocyte sedimentation rate (ESR) and Creative protein (CRP) levels, another consistent finding. Imaging modalities used include plain radiographs, computed tomography (CT) scan and magnetic resonance imaging (MRI). Findings include discitis, paraspinal and prevertebral abscesses, collapse and compression.

Radiological findings are often non-specific, thus nondiagnostic and fail to provide a prognostic perspective if the disease is established. Therefore, a bacterial and/or a histological evidence must be obtained to distinguish one infection from another, or from a neoplasm [10]. Localization using percutaneous CT-guided biopsy, introduced in the 1970s, is believed to be the single most accurate method for performing biopsies [11,12] and is now routinely used in the diagnosis of spinal lesions [13-18].

Due to the lack of image guided biopsy centers and the high prevalence of the disease, most of the patients are diagnosed and treated on clinical grounds. This practice was thought to lead to a delay in the diagnosis of other pathologies and MDR TB. The Aga Khan University Hospital is one of the few centers where percutaneous CT-guided biopsy is available. We therefore decided to evaluate our use of CT-guided biopsy and its effect on the management of Potts disease at our center.

\section{Materials and Methods}

This was a retrospective observational study conducted at the Neurosurgery section of Aga Khan University Hospital (AKUH) Karachi. AKUH is a Joint Commission of International Accreditation accredited, International
Organization for Standardization) certified, tertiary care hospital with over 43 specialties. Potts disease is treated by a multidisciplinary team consisting of members from Neurosurgery, Infectious Diseases and Radiology Departments. The duration of this study was 7 years (2007-2013). The review of medical records was done over three months (January to March, 2014).

\section{Patient selection}

All the patients with suspected Potts disease who underwent CT-guided biopsy at the Aga Khan University Hospital during the study period were included in the study.

\section{Technique}

Radiology of the patients was reviewed by consultant radiologists for feasibility of the procedure. Patients with lesions located anterior to the cord or vertebral bodies did not undergo the procedure. The procedures were performed by 4 different Interventionists using similar techniques as described below. The biopsy route was planned to avoid neurovascular bundles and uninvolved tissue compartments.

The procedure was mostly performed in the prone position. Short bursts of intermittent CT fluoroscopic screening were used to reduce radiation dosage to the patient. Following preliminary axial CT scanning, the most appropriate slice was selected for directing the needle into the lesion. In the case of multiple lesions, the largest and most superficial lesion was chosen. Any soft tissue mass related to the bony lesion was also biopsied and aspirated whenever possible.

\section{Data collection}

The medical records of the selected patients were pulled out of the medical records room. A pro forma was developed and pre tested before final data collection. The pro forma included such variables as age, gender, symptoms and their duration as well as radiological and clinical features of the disease. Details of the procedure, its complications and information on the requested microbiology and histopathology were also part of the pro forma. We retrieved the radiological images of all patients through Hospital Patient Inquiry to record the radiological features at presentation. We used the same software to record 
serological markers of ESR and CRP. We pilot tested the pro forma for correct recording of variables. Hospital records were consulted for treatment and follow up progress. We used SPSS ver. 17 (SPSS Inc., Chicago, IL, USA) to record all the data.

\section{Data analysis}

We analyzed the data using SPSS ver. 17 (SPSS Inc.). Continuous variables were expressed using means and standard deviations. Categorical variables were expressed using percentages and proportions.

\section{Results}

178 patients were treated for suspected Potts disease during the study period. CT-guided biopsies of the spinal lesions were performed in 91 patients $(51.12 \%)$. There was no preponderance of either gender (Table 1). Majority of the patients presented with back pain and had significant elevations of ESR and CRP (Table 1). Other demographic and clinical characteristics of patients are given in Table 1.

On imaging, 38 patients (41.75\%) had abscesses and 7 (7.69\%) had mass lesions (Table 1).

Of the 91 procedures, $22(24.2 \%)$ were inconclusive because of inadequate sample (10), normal tissue (6) or reactive tissue (6). 69 biopsies came back positive (75.8\%) (Fig. 1). The results of the procedure are presented in the flow diagram in Fig. 1.

We had a mean follow up of $14.7 \pm 7.8$ months. The response to antituberculosis therapy (ATT) was determined by the infectious disease team on the basis of ESR, CRP, clinical and radiological features. All 91 cases in which CT-guided biopsy was performed responded positively to ATT. Among the remaining 87 cases in which CT-guided biopsy was not performed, 80 cases responded positively to ATT.

Only 2 patients $(2.1 \%)$ had a hematoma at the procedure site.

\section{Discussion}

Potts disease is diagnosed on the basis of clinical and radiological data [19]. We treated $49 \%$ of the patients without biopsy in this series. But with the emergence of resistant strains it is being recommended that a biopsy be performed whenever possible. Diagnostic yields of
Table 1. Demographics and clinical characteristics

\begin{tabular}{lc}
\hline Characteristic & Variable \\
\hline Age (yr) & $45.01 \pm 20.99$ \\
\hline Gender & \\
\hline Male & $49(54)$ \\
\hline Female & $42(46)$ \\
\hline Presentation & \\
\hline Back pain & $66(72.5)$ \\
\hline Neurological deficit & $30(33.0)$ \\
\hline Fever & $16(17.6)$ \\
\hline Weight loss & $38(41.7)$ \\
\hline Co-morbids & \\
\hline Hypertension & $18(19.8)$ \\
\hline Diabetes mellitus & $15(16.5)$ \\
\hline Radiological characteristics & \\
\hline Abscess & $38(41.75)$ \\
\hline Vertebral collapse & $29(31.8)$ \\
\hline Mass lesion & $7(7.69)$ \\
\hline Compression fracture with cord compression & $7(7.69)$ \\
\hline Serology & $16(17.58)$ \\
\hline Erythrocyte sedimentation rate & $28(30.76)$ \\
\hline C-reative protein & $42.96 \pm 30.79$ \\
\hline History of exposure & $6.39 \pm 6.62$ \\
\hline
\end{tabular}

Values are presented as mean \pm standard deviation or number (\%).

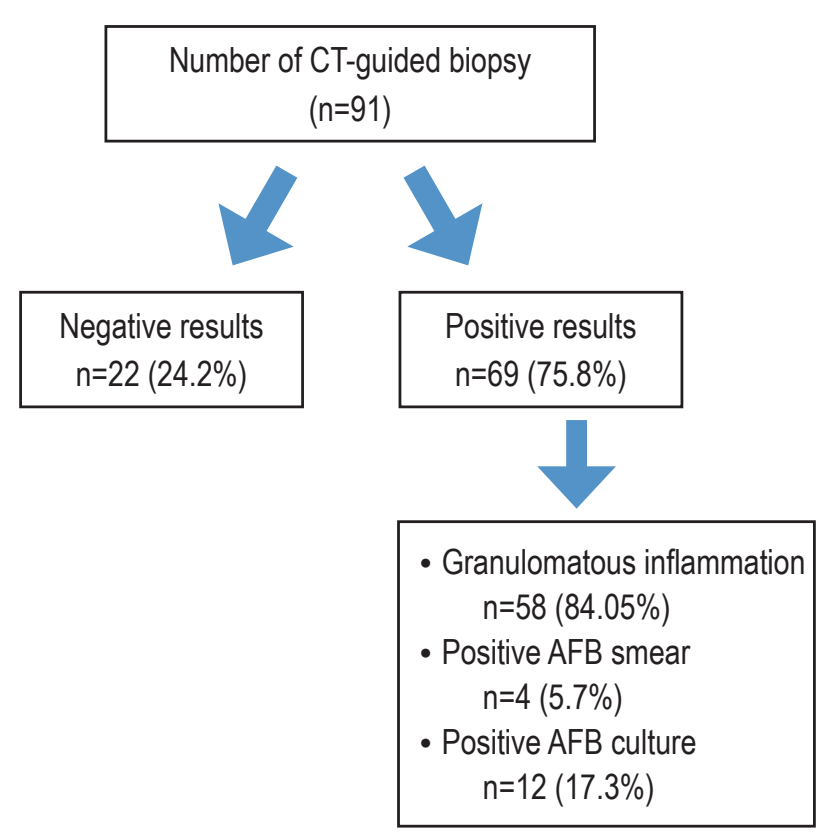

Fig. 1. Flow chart showing patient selection and results. CT, computed tomography; AFB, acid fast bacilli. 
CT scan guided biopsies for Potts disease differ. We got a positive answer in $75.8 \%$ of all the cases where a CTguided biopsy was performed. However all the patients with a positive diagnosis had Potts disease. Francis et al. [20] reported the best results in literature so far, (82\%) but their study had a comparatively small sample size of 29. The discrepancy in success rates has been attributed to accessibility of the lesion, the operator's technique and experience and the number of microbacteria and Langarhan giant cells in the specimen (Sp. Nature) [21]. The current study is the largest case series assessing the diagnostic value of procedure. In a systematic review, Colmenero et al. [19] reported positive histopathology results ranging from $29 \%$ to $100 \%$.

Positive acid-fast bacillus (AFB) cultures were seen in $13.2 \%$ of cases. The percentage of positive AFB smear was $4.4 \%$. These values are the lowest in reported literature. The percentage of positive AFB cultures ranged from $50 \%$ to $83 \%$ with an average of $66.2 \%$ and the percentage of positive AFB smears on Potts disease samples ranged from $30 \%$ to $57.5 \%$ with an average of $37 \%$ [19].

As noted above, the discrepancy in the success rate is probably due to the accessibility of the lesion, the operator's technique and experience and the number of microbacteria and Langham giant cells in the specimen. Both microbiology and histopathology are recommended on tissue samples obtained through CT-guided biopsy. Bacillary population is lower in samples of tuberculosis obtained from extra pulmonary samples when compared to pulmonary disease [19]. Micrscopic examinations for Mycobacterium tuberculosis were positive $36 \%$ of the cases $[19,22]$ or less. Our study had the lowest number of positive microscopic examinations (4.4\%). This raises the question as to whether every sample obtained through CT-guided biopsy should be sent for microscopic examination.

All the patients who had positive histopathological and microbiological results responded to ATT. Although all granulomas are not tuberculosis, the patients who were treated with ATT responded well.

The rate of complications in CT-guided biopsy for spinal lesions vary from $0 \%$ to $10 \%$. In our study two patients had local hematoma that was managed conservatively. Every patient was screened for any coagulation derangement by a prothrombin time and activated partial thromboplastin time. The procedure was not performed on patients with deranged coagulation parameters.
The study however has several limitations. It was a retrospective study with an inherent bias of patient selection. Lesions were heterogeneous in location and had diverse clinical presentations. The procedures were performed by different interventionists and different pathologists were involved. However, the study presents 7 year results from an established neurosurgical center in an endemic area. The results are comparable to available data and encourage the use of CT-guided biopsy.

\section{Conclusions}

The use of CT-guided biopsy in the diagnosis of Potts disease is increasing. We got a positive answer in $75.8 \%$ of cases. Granulomatous inflammation on histopathology was the commonest diagnostic feature with a comparatively low rate of positive AFB smear and culture.

\section{Conflict of Interest}

No potential conflict of interest relevant to this article was reported.

\section{References}

1. World Health Organization (WHO). Pakistan: stop tuberculosis [Internet]. Islamabad: WHO; 2014 [cited 2015 Apr 22]. Available from: http://www.emro.who. int/pak/programmes/stop-tuberculosis.html.

2. Miandad M, Burke F, Nawaz-ul-Huda S, Azam M. Tuberculosis incidence in Karachi: a spatio-temporal analysis. Malays J Soc Space 2014;10(5):1-8.

3. Gautam MP, Karki P, Rijal S, Singh R. Pott's spine and paraplegia. JNMA J Nepal Med Assoc 2005;44:10615.

4. Moon MS. Tuberculosis of the spine: controversies and a new challenge. Spine (Phila Pa 1976) 1997;22: 1791-7.

5. Wang H, Li C, Wang J, Zhang Z, Zhou Y. Characteristics of patients with spinal tuberculosis: seven-year experience of a teaching hospital in Southwest China. Int Orthop 2012;36:1429-34.

6. Luk KD. Tuberculosis of the spine in the new millennium. Eur Spine J 1999;8:338-45.

7. McLain RF, Isada C. Spinal tuberculosis deserves a place on the radar screen. Cleve Clin J Med 2004;71: 537-9. 
8. Le Page L, Feydy A, Rillardon L, et al. Spinal tuberculosis: a longitudinal study with clinical, laboratory, and imaging outcomes. Semin Arthritis Rheum 2006; 36:124-9.

9. Mulleman D, Mammou S, Griffoul I, Avimadje A, Goupille P, Valat JP. Characteristics of patients with spinal tuberculosis in a French teaching hospital. Joint Bone Spine 2006;73:424-7.

10. Ahmadi J, Bajaj A, Destian S, Segall HD, Zee CS. Spinal tuberculosis: atypical observations at MR imaging. Radiology 1993;189:489-93.

11. Adapon BD, Legada BD Jr, Lim EV, Silao JV Jr, Dalmacio-Cruz A. CT-guided closed biopsy of the spine. J Comput Assist Tomogr 1981;5:73-8.

12. Haaga JR, Alfidi RJ. Precise biopsy localization by computer tomography. Radiology 1976;118:603-7.

13. Babu NV, Titus VT, Chittaranjan S, Abraham G, Prem H, Korula RJ. Computed tomographically guided biopsy of the spine. Spine (Phila $\mathrm{Pa} 1976$ ) 1994;19:2436-42.

14. Brugieres P, Revel MP, Dumas JL, Heran F, Voisin MC, Gaston A. CT-guided vertebral biopsy: a report of 89 cases. J Neuroradiol 1991;18:351-9.

15. Cleary K, Clifford M, Stoianovici D, Freedman M, Mun SK, Watson V. Technology improvements for image-guided and minimally invasive spine procedures. IEEE Trans Inf Technol Biomed 2002;6:249-
61.

16. Dupuy DE, Rosenberg AE, Punyaratabandhu T, Tan $\mathrm{MH}$, Mankin HJ. Accuracy of CT-guided needle biopsy of musculoskeletal neoplasms. AJR Am J Roentgenol 1998;171:759-62.

17. Kang M, Gupta S, Khandelwal N, Shankar S, Gulati M, Suri S. CT-guided fine-needle aspiration biopsy of spinal lesions. Acta Radiol 1999;40:474-8.

18. Kattapuram SV, Rosenthal DI. Percutaneous biopsy of the cervical spine using CT guidance. AJR Am J Roentgenol 1987;149:539-41.

19. Colmenero JD, Ruiz-Mesa JD, Sanjuan-Jimenez R, Sobrino B, Morata P. Establishing the diagnosis of tuberculous vertebral osteomyelitis. Eur Spine J 2013;22 Suppl 4:579-86.

20. Francis IM, Das DK, Luthra UK, Sheikh Z, Sheikh M, Bashir M. Value of radiologically guided fine needle aspiration cytology (FNAC) in the diagnosis of spinal tuberculosis: a study of 29 cases. Cytopathology 1999; 10:390-401.

21. Wang D. Diagnosis of tuberculous vertebral osteomyelitis (TVO) in a developed country and literature review. Spinal Cord 2005;43:531-42.

22. Pertuiset E, Beaudreuil J, Liote F, et al. Spinal tuberculosis in adults: a study of 103 cases in a developed country, 1980-1994. Medicine (Baltimore) 1999;78: 309-20. 